\title{
REMARKS ON TRANSITIVITIES OF BETWEENNESS
}

W. R. TRANSUE

This note provides lattice theoretic interpretations of the transitivities

$$
\begin{aligned}
& T_{8 \cdot} a b c \cdot d a b \cdot x c d \cdot a \neq b \rightarrow a c x, \\
& T_{9 \cdot} a b c \cdot d a b \cdot x c d \cdot a \neq b \rightarrow b c x, \\
& T_{10} \cdot a b c \cdot a b d \cdot x b c \cdot a \neq b \cdot b \neq c \rightarrow x b d,
\end{aligned}
$$

introduced by Pitcher and Smiley. ${ }^{1}$ It may be recalled that in a lattice the relation $a b c$ ( $b$ is between $a$ and $c$ ) is said to hold if and only if

$$
(a \cup b) \cap(b \cup c)=b=(a \cap b) \cup(b \cap c) .
$$

THEOREM 1. If $L$ is a lattice then its betweenness relation has one of the transitivities $T_{8}$ or $T_{9}$ if and only if $L$ is linearly ordered.

Proof. It is obvious that $T_{8}$ and $T_{9}$ are satisfied if $L$ is linearly ordered. To show that $T_{8}$ implies linear order, consider two elements $a, c \in L$. Suppose that $a$ and $c$ are not comparable, that is, that none of the relations $a=c, a\langle c, a\rangle c$ holds. Then $a \neq a \cup c, c \neq a \cup c$. Moreover, we have

$$
a a \cup c c \cdot a \cap c a a \cup c \cdot a \cup c c a \cap c \cdot a \neq a \cup c
$$

and by $T_{8}$ this implies $a c a \cup_{c}$ which, with $a a \cup_{c} c$, implies $c=a \cup_{c}$, contrary to our assumption that $c \neq a \cup c$. In the same way $T_{9}$ can be shown to imply linear order.

THEOREM 2. If $L$ is a lattice then its betweenness relation has the transitivity $T_{10}$ if and only if $L$ is linearly ordered or is composed of two linearly ordered systems with a common greatest element, I, and a common least element, 0 .

Proof. It is easy to see that lattice betweenness in such a lattice has the transitivity $T_{10}$. Denote the two linearly ordered systems by $L_{1}$ and $L_{2}$. Then if, in the hypotheses of $T_{10}, b \neq 0, b \neq I, b \in L_{1}$, all the elements $a, c, d$, and $x$ must belong to $L_{1}$ and the conclusion follows from the fact that $T_{10}$ holds for linear order. If $b=0$ or $b=I$ in the hypotheses of $T_{10}$ and if $a \in L_{1}$, then we must have $c \in L_{2}, d \in L_{2}$, $x \in L_{1}$ and the conclusion again follows.

Received by the editors February 26, 1943.

1 Everett Pitcher and M. F. Smiley, Transitivities of betweenness, Trans. Amer. Math. Soc. vol. 52 (1942) pp. 95-114. We shall use the notations and terminology of Pitcher and Smiley. 
To show that $T_{10}$ implies that the lattice must have one of these forms, we show first that the lattice consists of a number of chains connecting 0 and $I$ unless it is linearly ordered and then show that the number of these chains cannot be greater than two. To prove the first of these statements let us show that if $a$ and $b$ are two elements which are not comparable then there is a greatest element, $I$, and a least element, 0 , and $a \cup b=I, a \cap b=0$. Suppose $a \cup b \neq I$, that is, that $a \cup b \geqq x$ for every $x \in L$ fails. Then there exists $u \in L$ such that $u>a \cup b$ and

$$
a a \cup b u \cdot a a \cup b b \cdot b a \cup b u \cdot a \neq a \cup b \cdot a \cup b \neq u \rightarrow b a \cup b b
$$

which implies $a \cup b=b$ and $b \geqq a$, contrary to the assumption that $a$ and $b$ are not comparable. This shows that $a \cup b=I$, and $a \cap b=0$ may be shown in the same way.

To show that the number of chains is not greater than two we again suppose the contrary. Then we have three elements $a, b$, and $c$, no two of which are comparable. Then from $T_{10}$, since $a \cup b=a \cup c$ $=b \cup c=I$,

$$
a I b \cdot a I c \cdot c I b \cdot a \neq I \cdot I \neq b \rightarrow c I c
$$

which implies $c=I$, contrary to our assumption that it was not comparable with $a$ and $b$. This completes the proof of Theorem 2 .

Finally we include a correction to the previously quoted paper of Pitcher and Smiley. ${ }^{2}$ It is stated (p. 113) that "Examples of semi metric spaces are easily given in which $\tau_{2}$ fails."

$$
\text { ז. } a b c \cdot a d b \cdot d b c \rightarrow a d c .
$$

That this transitivity is, in fact, present in all semi metric spaces may be seen as follows. The hypotheses of $\tau_{2}$ for betweenness in a semi metric space are

$$
\begin{aligned}
& \delta(a, b)+\delta(b, c)=\delta(a, c), \\
& \delta(a, d)+\delta(d, b)=\delta(a, b), \\
& \delta(d, b)+\delta(b, c)=\delta(d, c)
\end{aligned}
$$

Substituting from (2) in (1) and using (3) we have $\delta(a, d)+\delta(d, c)$ $=\delta(a, c)$. A consequence of this fact is that no non-modular lattice can be supplied with a semi metric so that metric and lattice betweenness are the same.

\section{INSTITUTE FOR ADVANCEd STUdy}

2 This error was noticed by the authors and is included here at their request. 